\title{
Graphene oxide-multiwalled carbon nanotubes composite as an anode for lithium ion batteries
}

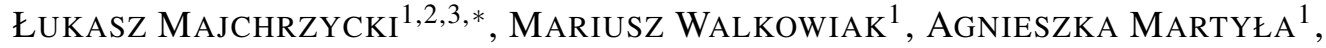 \\ MiKHAIL Y. YABLOKOV ${ }^{4}$, MAREK NOWICKI ${ }^{2}$, RYSZARD CZAJKA $^{2}$ \\ ${ }^{1}$ Institute of Non-Ferrous Metals, Poznań Branch, Central Laboratory of Batteries and Cells, \\ Forteczna 12, 61-362 Poznań, Poland \\ ${ }^{2}$ Institute of Physics, Poznań University of Technology, Piotrowo 3, 60-965 Poznań, Poland \\ ${ }^{3}$ Wielkopolska Center for Advanced Technologies, Umultowska 89C, 61-614 Poznań, Poland \\ ${ }^{4}$ Russian Academy of Sciences, Enikolopov Institute of Synthetic Polymer Materials, \\ 70 Profsoyuznaya St., 117393 Moscow, Russia
}

\begin{abstract}
Nowadays reduced graphene oxide (rGO) is regarded as a highly interesting material which is appropriate for possible applications in electrochemistry, especially in lithium-ion batteries (LIBs). Several methods were proposed for the preparation of rGO-based electrodes, resulting in high-capacity LIBs anodes. However, the mechanism of lithium storage in rGO and related materials is still not well understood. In this work we focused on the proposed mechanism of favorable bonding sites induced by additional functionalities attached to the graphene planes. This mechanism might increase the capacity of electrodes. In order to verify this hypothesis the composite of non-reduced graphene oxide (GO) with multiwalled carbon nanotubes electrodes was fabricated. Electrochemical properties of GO composite anodes were studied in comparison with similarly prepared electrodes based on rGO. This allowed us to estimate the impact of functional groups on the reversible capacity changes. As a result, it was shown that oxygen containing functional groups of GO do not create, in noticeable way, additional active sites for the electrochemical reactions of lithium storage, contrary to what has been postulated previously.
\end{abstract}

Keywords: graphene oxide; reduced graphene oxide; Li-ion battery; composite anode; porous electrode

(C) Wroclaw University of Technology.

\section{Introduction}

Carbon materials are a very broad and popular group of active anode materials for lithium-ion batteries (LIBs) technology. They have been investigated for more than 20 years and still are developed [1]. Although the time of great interest in research on graphite electrodes has gone, recently there has been a considerable interest in another allotrope of carbon called graphene and in its derivatives, especially reduced graphene oxide (rGO). As a kind of disordered carbon, rGO is a part of long history research on electrode materials such as hard and soft carbons, single- and multi-walled carbon nanotubes and related compounds [2,3]. Such

*E-mail: lukasz.majchrzycki@gmail.com materials often can provide great capacity in comparison with graphite. However, they commonly exhibit high hysteresis and irreversibility [4], which strongly limits their application.

Starting material to obtain rGO is graphene oxide (GO). It is one atom thick graphene layer decorated on the basal plane and at the edges by functionalities, especially epoxy, hydroxyl and carboxyl groups $[5,6]$. Thanks to variety of functionalities, it has completely different properties in comparison with graphene [7]. As a result of presence of oxygen-containing functional groups, graphene oxide becomes well soluble in water and other basic media [8]. Because of the vast amount of the carbon atoms with $\mathrm{sp}^{3}$ hybridization in comparison with $\mathrm{sp}^{2}$, graphene oxide also became insulating and has a large amount of defects in its atomic structure [7]. 
But the highly attractive property of GO is that it can be easily converted, by a variety of reduction processes, into rGO [9], which is a very promising material, especially for electrochemistry. Currently, rGO is strongly investigated for the usage in LIBs either as an additive for developing electrical contact [10] or an aid during the synthesis of electrode material [11] as well as an electrode active material [12-16]. Pure rGO used as LIBs anode exhibits high capacity, few times higher than graphite. There is also noticeable that the capacity of thermally reduced GO is usually higher than that of hydrazine. In the first cycle the reversible specific capacity of once oxidized GO after thermal annealing reduction can achieve $1264 \mathrm{mAh} \cdot \mathrm{g}^{-1}$ [12], while the three times oxidized annealed GO capacity can be as high as $2311 \mathrm{mAh} \cdot \mathrm{g}^{-1}$. That is more than six times higher than capacity of graphite [13].

The main purpose of this study is to analyze the impact of the oxygen-containing functional groups on high excess of capacity of rGO anodes over the graphite ones. This is the one of suggested, but not the only possible mechanism of additional lithium storage [1]. The other possible scenario assumes that the additional capacity can be also the result of high degree disorder of rGO [14]. Moreover, other mechanisms, like micro-cavities lithium storage or surface storage are taken into consideration $[1,17]$. The reason of excess of the capacity is still the matter of intensive studies and discussions. The understanding of the nature of lithium storage into rGO can contribute to improvement of the properties of this very promising candidate for a LIB's high capacity anodes. However, to determine the mechanism of excess of the capacity a model system is required. So far, there are no experimental results of the electrochemical lithium storage in a nonreduced GO. Here we try to fill this gap in order to examine the influence of the functional groups on the reversible capacity of GO and rGO. It has been done thanks to preparation of porous composite electrodes consisting of GO and multiwalled carbon nanotubes (MWNTs). Such electrodes fulfill the requirements for electrodes: good electrical contact, good electrolyte access and high specific surface area. The composite electrodes were reduced by the photothermal method [18] for a partial removal of functional groups what allowed to analyze their influence on capacity of anode. Furthermore, GO here acts as a binder [19], what highly increases mechanical stability of the electrodes.

\section{Experimental}

\subsection{Preparation of GO/MWNTs compo- site electrodes}

Graphene oxide (GO) was obtained from natural graphite by modified Hummers method [20] as it was described before [21]. Composite electrodes with typically $9 \%$ of MWNTs were prepared by the direct mixing of GO and MWNTs in N-methylpyrrolidone (NMP) as it was described previously [22]. In details, $60 \mathrm{~mL}$ of GO suspension in water with a concentration of $1 \mathrm{mg} / \mathrm{mL}$ were centrifuged at $15000 \mathrm{rpm}$ for $70 \mathrm{~min}$. The solution over the precipitate was removed. Preserved precipitate was added to $6 \mathrm{mg}$ of MWNTs (Sigma Aldrich, diameter $110 \mathrm{~nm}$ to $170 \mathrm{~nm}$, length $5 \mu \mathrm{m}$ to $9 \mu \mathrm{m}$, purity $90 \%$ ) suspended in $3 \mathrm{~mL}$ of NMP. The suspension was stirred and heated up to $150{ }^{\circ} \mathrm{C}$ until a dense slurry was obtained. The slurry was mixed, dispersed on a $12 \mathrm{~mm}$ copper foil discs and then vacuum dried at $120{ }^{\circ} \mathrm{C}$ overnight. The mass of electrode was approximately $1 \mathrm{mg}$. Some of such prepared electrodes were then reduced by the photothermal method [17]. This process was carried out in air atmosphere by focusing a camera photoflash lamp on the composite GO/MWNTs electrode. Distance between the flash and electrode was $\sim 1 \mathrm{~cm}$. Photothermal process was carried out 10 times to ensure complete reduction.

\subsection{Characterization}

The structure of obtained electrodes was characterized by X-ray powder diffraction (XRD) with a Siemens D500 diffractometer with a $\mathrm{CuK} \alpha$ radiation $(\lambda=1.542 \AA)$ source. The morphology of the materials was examined by scanning electron microscopy (SEM) Zeiss EVO 40. The wavelength-dispersive X-ray spectroscopy (WDS) was performed using Quanta FEG 250 (FEI) SEM equipped with low energy X-ray 
spectrometer LEXS (EDAX) to analyze samples chemical composition.

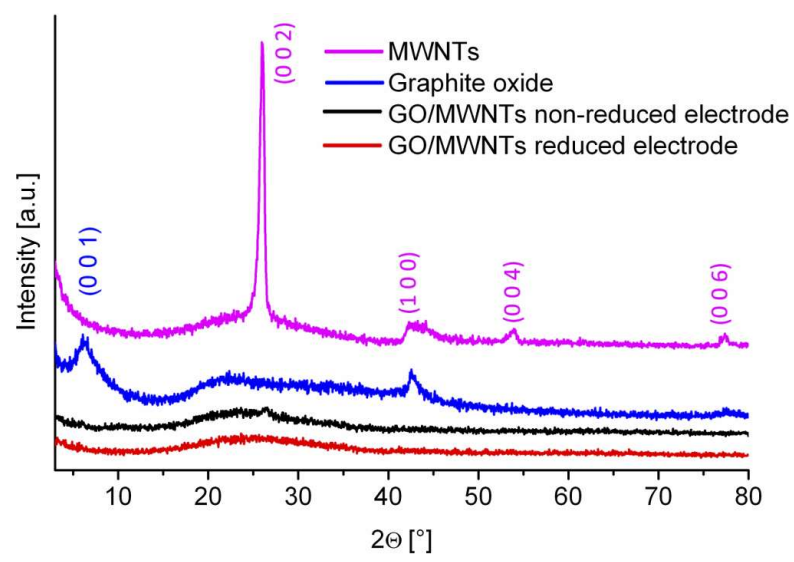

Fig. 1. XRD patterns of electrode materials, MWNTs and graphite oxide.

The galvanostatic charging/discharging and cyclic voltammetry experiments were carried out using a two-electrode Swagelok ${ }^{\circledR}$ type electrochemical cell with a lithium foil as a counter and reference electrodes. An LP30 $(1 \mathrm{M} \mathrm{LiPF} 6$ in ethylene carbonate: dimethyl carbonate 1:1) electrolyte (Merck) was used. All the electrodes, prepared exactly as described above, were tested without using any additional binders or conductive additives. The assembly process was carried out in argon atmosphere in a glove box with humidity less than $1 \mathrm{ppm}$. The galvanostatic charge/discharge measurements were carried out in a voltage range of $0.0 \mathrm{~V}$ to $3.0 \mathrm{~V}$ with a current densities of $10 \mathrm{mAh} \cdot \mathrm{g}^{-1}, 30 \mathrm{mAh} \cdot \mathrm{g}^{-1}, 50 \mathrm{mAh} \cdot \mathrm{g}^{-1}$ and $100 \mathrm{mAh} \cdot \mathrm{g}^{-1}$. Current density and capacity values were calculated for the mass of GO taken for electrode preparation. The cyclic voltammetry measurements were performed in the same voltage range like galvanostatic experiments, with a scan rate of $0.02 \mathrm{mV} \cdot \mathrm{s}^{-1}$. All electrochemical experiments were done using a VMP3 modular 16 channel potentiostat/galvanostat (Bio-Logic).

\section{Results and discussion}

\subsection{Structure and morphology}

Fig. 1 illustrates XRD patterns of electrode materials, as well as their components: graphite oxide and MWNTs. In the structure of MWNTs pattern sharp peak at the angle $2 \theta$ of $25.96^{\circ}$ can be found, which can be indexed as the diffraction pattern of its $\left(\begin{array}{lll}0 & 0 & 2\end{array}\right)$ crystal face [23]. High angle diffraction peaks observed at Bragg angels of $43.4^{\circ}, 53.8^{\circ}$ and $77.3^{\circ}$ can be matched to crystal faces $\left(\begin{array}{lll}1 & 0 & 0\end{array}\right),\left(\begin{array}{lll}0 & 0 & 4\end{array}\right)$ and $\left(\begin{array}{lll}0 & 0 & 6\end{array}\right)$, respectively. Graphite oxide pattern exhibits peaks at the $2 \theta$ angle of $6.2^{\circ}$ and $42.7^{\circ}$ which could be identified as the graphite oxide $\left(\begin{array}{lll}0 & 0 & 1\end{array}\right)$ and $\left(\begin{array}{lll}1 & 0 & 0\end{array}\right)$ crystal faces, respectively [24]. At the GO/MWNTs composite electrode no clear diffraction peaks are seen. This suggests presence of high amount of disordered carbon planes which come from exfoliated GO and thus it does not exhibit the $\left(\begin{array}{lll}0 & 0 & 1\end{array}\right)$ diffraction peak of graphite oxide because of its exfoliation. Only a small diffraction peak coming from the $\left(\begin{array}{lll}0 & 0 & 2\end{array}\right)$ MWNTs crystal face is slightly seen. Besides, the reduction process has not changed the XRD pattern noticeably.

In Fig. 2, the cross-section SEM images of electrode materials are shown. Fig. 2a presents low magnification of non-reduced electrode. Fig. 2b and Fig. 2c show higher magnifications of nonreduced and photothermally reduced electrodes, respectively. These SEM images, as well as the XRD patterns, confirm the high degree of disorder between GO planes. As shown in the Fig. 2b, due to presence of MWNTs between GO layers, even electrodes before the reduction process exhibited porous structure. That is appropriate in the case of good electrolyte access. Such porosity could be the great advantage of our composite electrodes in comparison with the previous work [17], where the porous structure was induced by rapid outgassing of GO during the photothermal reduction process resulting in GO exfoliation. However, in our experiment, high electrode porosity is noticeable also for non-reduced electrode material. Hence, in spite of evident degassing of the sample during the reduction process, the exfoliation is highly limited because of good gas escape. Therefore the structure of reduced electrode, shown in Fig. 2c, is only slightly expanded in comparison to the non-reduced one.

In order to analyze the effect of reduction on the elemental composition, WDS measurements were carried out. In Fig. 3, the WDS spectrum 


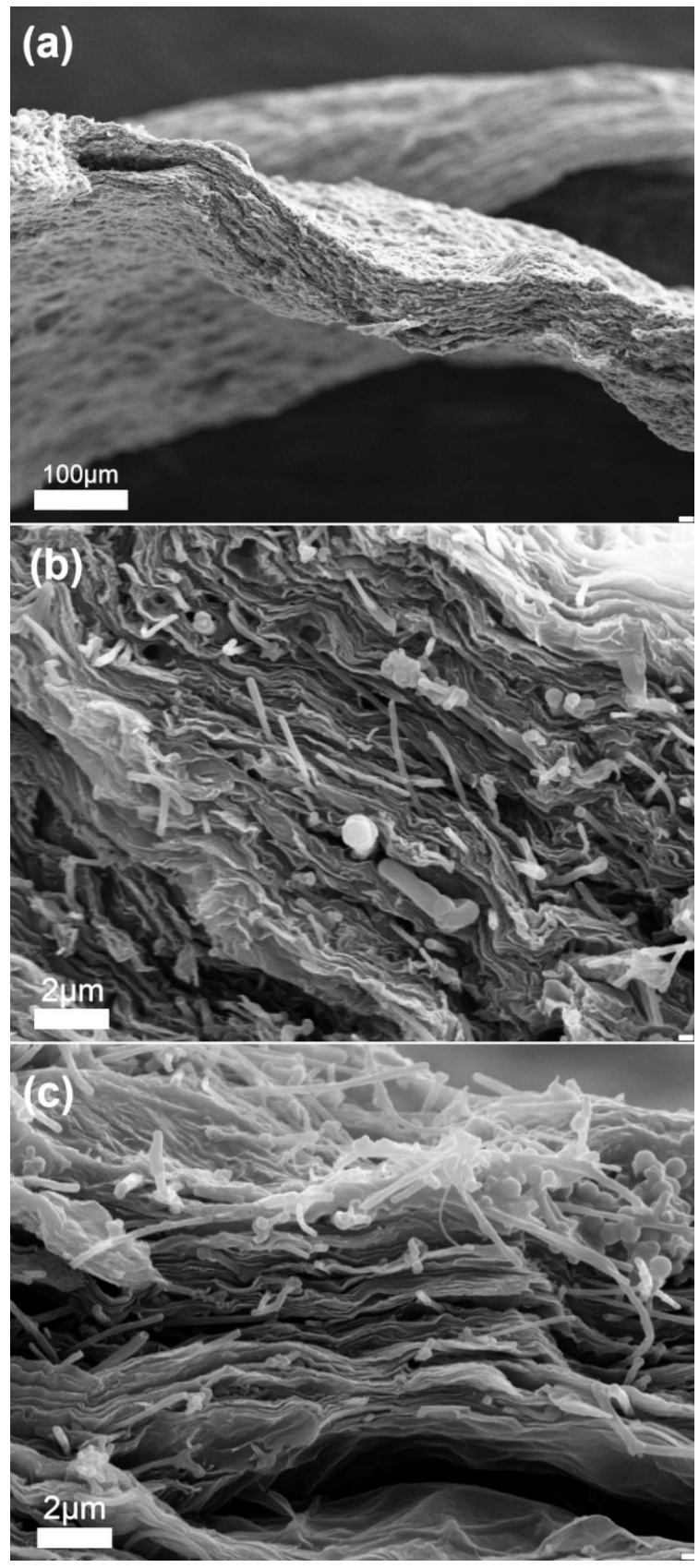

Fig. 2. Cross-section SEM images of non-reduced electrode $(\mathrm{a}, \mathrm{b})$ and photothermally reduced (c) electrodes.

of GO/MWNTs non-reduced electrode is shown. There are a clearly visible intense peak of $\mathrm{CK} \alpha$ line and two lower ones of $\mathrm{NK} \alpha$ and $\mathrm{OK} \alpha$. The percentage quantifications of carbon, nitrogen and oxygen in the electrodes before and after the reduction processes are presented in Table 1. As it is seen,

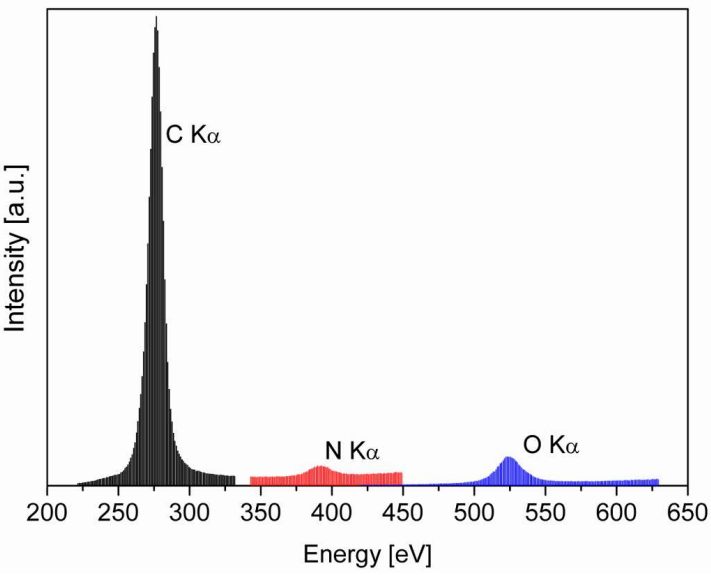

Fig. 3. WDS spectrum of GO/MWNTs non-reduced electrode.

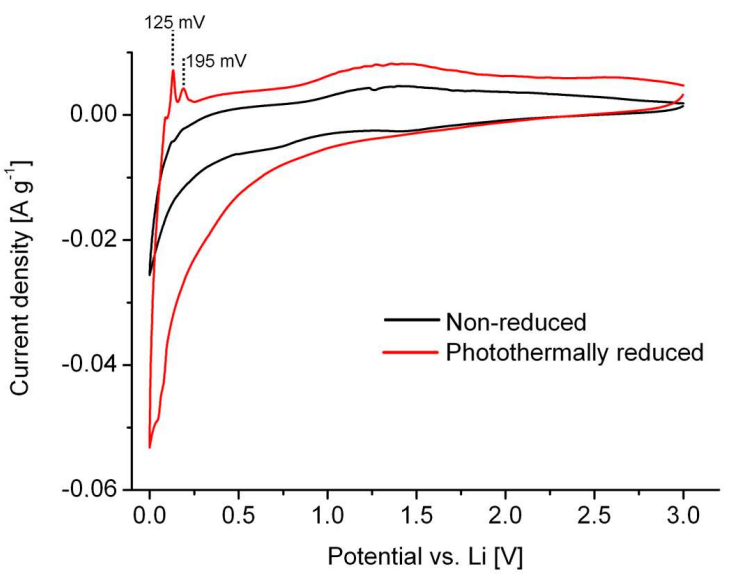

Fig. 4. Cyclic voltammetric curves of electrodes.

the reduction process has increased the amount of carbon by about $3 \%$ with respect to the amount of nitrogen. Moreover, the sum of air components is still quite high after the reduction process, which suggests that such kind of reduction process is not as effective as e.g. some other complex, multi-step strategies [25].

Table 1. Composition of the non- and photothermally reduced electrodes using the WDS.

\begin{tabular}{lcc}
\hline & Non-reduced & Photothermally reduced \\
\hline \hline $\mathrm{CK} \alpha[\%]$ & $80.63 \pm 0.17$ & $83.53 \pm 0.27$ \\
$\mathrm{NK} \alpha[\%]$ & $8.3 \pm 1.2$ & $5 \pm 3$ \\
$\mathrm{OK} \alpha[\%]$ & $11.1 \pm 0.6$ & $11 \pm 1$ \\
\hline
\end{tabular}


(a)

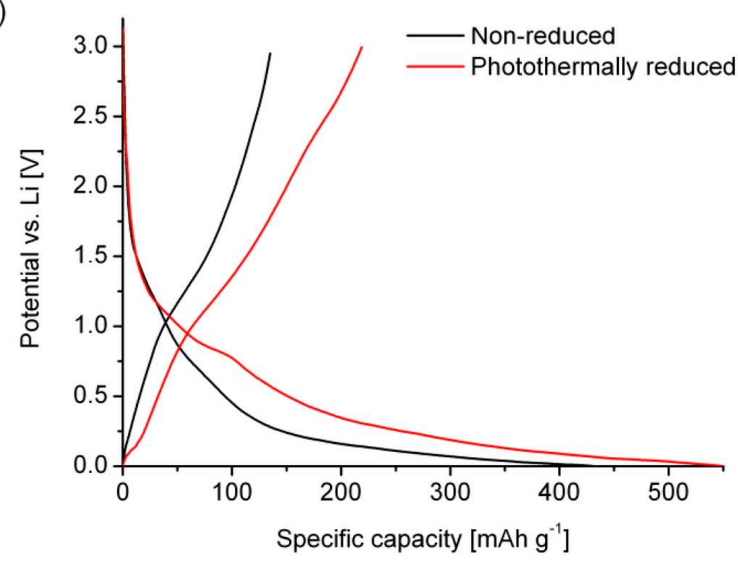

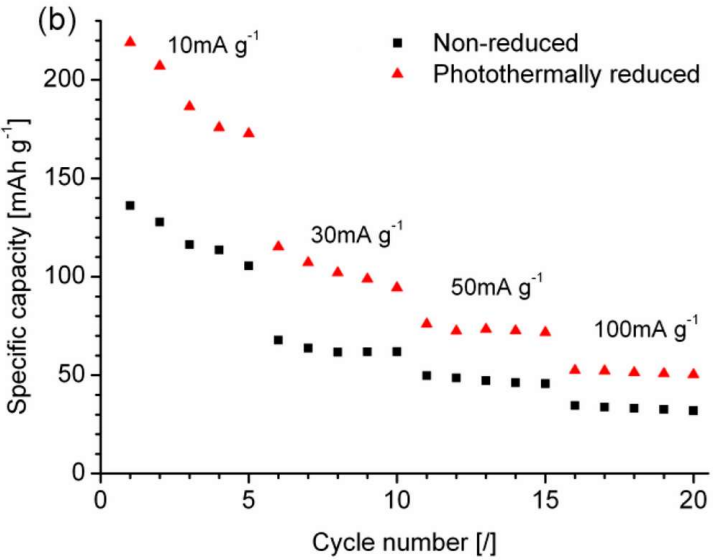

Fig. 5. First cycle charge/discharge profiles (a) and specific capacity of GO/MWNTs electrodes (b).

\subsection{Electrochemical performance}

The first loop of cyclic voltammetric curves for non-reduced GO/MWNTs and photothermally reduced electrodes are shown in Fig. 4. For the nonreduced electrode, the cyclic voltammogram does not show any distinct peaks, which is characteristic of disorder carbons. Reduction process leads to appearance of the intercalation peaks. The oxidative peak maxima are located at $125 \mathrm{mV}$ and $195 \mathrm{mV}$ for reduced electrode. They are slightly shifted in comparison to graphite intercalation peaks. The appearance of intercalation peaks suggests partial graphitization of the electrode materials.

In Fig. 5a, the first cycle charge/discharge profiles of electrodes are shown. There is clearly seen high irreversible capacity. Fig. 5b shows the cycling performances of electrodes at different current densities of $10 \mathrm{~mA} \cdot \mathrm{g}^{-1}, 30 \mathrm{~mA} \cdot \mathrm{g}^{-1}$, $50 \mathrm{~mA} \cdot \mathrm{g}^{-1}$ and $100 \mathrm{~mA} \cdot \mathrm{g}^{-1}$. The first cycle reversible capacity of non-reduced electrode at the current density of $10 \mathrm{~mA} \cdot \mathrm{g}^{-1}$ equals $136 \mathrm{mAh} \cdot \mathrm{g}^{-1}$, but such capacity rapidly decreases to $105 \mathrm{mAh} \cdot \mathrm{g}^{-1}$ just in $5^{\text {th }}$ cycle. Such rapid decreasing of the capacity is a typical behavior for all disordered carbons, especially rGO [11-15]. Higher current densities induce further capacity decreasing up to slightly above $30 \mathrm{mAh} \cdot \mathrm{g}^{-1}$ for current density of $100 \mathrm{~mA} \cdot \mathrm{g}^{-1}$. After the photothermal reduction, capacity of the electrode in first cycle achieves $219 \mathrm{~mA} \cdot \mathrm{g}^{-1}$, next it falls to $173 \mathrm{~mA} \cdot \mathrm{g}^{-1}$ in $5^{\text {th }}$ cycle at current density of $10 \mathrm{~mA} \cdot \mathrm{g}^{-1}$, and drops to $50 \mathrm{~mA} \cdot \mathrm{g}^{-1}$ for current density of $100 \mathrm{~mA} \cdot \mathrm{g}^{-1}$. Noticeable is, thus, an increase in capacity of more than $50 \%$ after the reduction process. However, in a previous work [17] the authors obtained for the photothermally reduced graphene oxide paper much higher capacities of an order of $550 \mathrm{~mA} \cdot \mathrm{g}^{-1}$. The explanation of such difference could be the much lower exfoliation of GO layers in our composite electrode during the rapid outgassing affected by reduction. It is highly probable that such diminished exfoliation is caused by the better gas escape realized by the porous electrode structure, what was also shown by SEM. That weakened the exfoliation of the electrode induced by the degassing process, what usually accompanies to reduction process. Consequently, it limits the increase of the capacity originating from higher specific surface area.

The second reason of rising the capacity after the reduction process is the creation of conductive pathways in GO [26]. It can also increase capacity thanks to the shorter path lengths for the electrons and higher electrode conductivity. Postulated elsewhere [1] creation of favorable bonding sites for lithium by the functional groups, should lead, on the contrary, to decrease in the capacity after reduction. Hence, impact of this, if exists, should be much less than that of two previous effects.

Finally, this leads to the conclusion that the GO reduction process can influence its capacity mainly 
by the changes in specific surface area of the electrodes and GO flakes conductivity. It leads to an increase of the capacity after reduction process. The opposite, capacity-enhancing effect, induced by the presence of the functional groups, has not been observed. This leads to the conclusion that oxidative functionalities do not create here additional active sites for electrochemical reactions of lithium storage.

\section{Conclusions}

In summary, we have successfully prepared GO/MWNTs composite porous electrodes and reduced them by the photothermal method. For the first time we measured the capacity of unmodified, non-reduced GO and compared it with similar but reduced electrodes. It was assumed that the non-reduced electrodes, thanks to higher content of functional groups, might offer more active sites for electrochemical reactions than the reduced electrodes. However, during this experiment the opposite effect appeared. In our investigation we observed a noticeable increase of the capacity, perhaps due to the creation of conductive pathways in the reduced electrodes and an increase of its specific surface area. That suggests only a slight influence of GO functional groups on the enhanced lithium storage in GO and $\mathrm{rGO}$.

\section{Acknowledgements}

The authors thanks the the Ministry of Science and Higher Education in Poland for financial support within the Project No. 06/62/DSPB/0216.

\section{References}

[1] Lahiri I., Choi W., Crit. Rev. Solid State Mater. Sci., 38 (2013), 128.

[2] KASKhedikAR N.A., MaIER J., Adv Mater., 21 (2009), 2664.

[3] Frąckowiak E., Béguin F., Carbon, 40 (2002), 1775.

[4] Béguin F., Chevallier F., Vix C., SaAdallah S., Rouzaud J.N., Frackowiak E., J. Phys. Chem. Solids, 65 (2004), 211.

[5] Eda G., ChHowalla M., Adv. Mater., 22 (2010), 2392.
[6] Loh K.P., Bao Q., Eda G., ChHowalla M., Nat. Chem., 2 (2010), 1015.

[7] Gengler R., Spyrou K., Rudolf P., J. Phys. D. Appl. Phys., 43 (2010), 374015.

[8] PARK S., RUOFF R.S., Nat. Nanotechnol., 4 (2009), 217.

[9] Pei S., Cheng H.-M., Carbon, 50 (2012), 3210.

[10] Brownson D., Kampouris D., Banks C., J. Power Sources, 196 (2011), 4873.

[11] MAJCHRZYCKI Ł., MichalsKA M., WALKOWIAK M., WilińsKi Z., LiPińsKA L., Pol. J. Chem. Technol., 15 (2013), 15.

[12] Yoo E.J., Kim J., Hosono E., ZHOU H.-S., Kudo T., Honma I., Nano Lett., 8 (2008), 2277.

[13] Lian P., Zhu X., Liang S., Li Z., Yang W., WANG H., Electrochim. Acta, 55 (2010), 3909.

[14] Lee W., Suzuki S., Miyayama M., Ceram. Int., 39 (2013), S753.

[15] Pan D., Wang S., Zhao B., Wu M, Zhang H, WANG Y., JiAO Z., Chem. Mater., 21 (2009), 3136.

[16] Wang G., Shen X., Yao J., Park J., Carbon, 47 (2009), 2049.

[17] Matsumura Y., Wang S., Mondori J., Carbon, 33 (1995), 1457.

[18] Mukherjee R., Thomas A.V., KrishnaMUrThy A., Koratkar N., ACS Nano, 6 (2012), 7867.

[19] Luo J., Tung V.C., Koltonow A.R., Jang H.D., Huang J., J. Mater. Chem., 22 (2012), 12993.

[20] Hummers W.S., Offeman R.E., J. Am. Chem. Soc., 80 (1958), 1339.

[21] MAJCHRZYCKI Ł., AUgustYNIAK-JABŁOKOW M.A., Strzelczyk R., MaćKowiak M., Acta Phys. Pol. A, 127 (2015), 540.

[22] Majchrzycki Ł., Walkowiak M., Patent Application PL 405725 (A1) (2013).

[23] Belin T., Epron F., Mater. Sci. Eng. B- Adv., 119 (2005), 105.

[24] Stankovich S., Dikin D.A., Piner R.D., KohlhaAs K.A., Kleinhammes A., JiA Y., Wu Y., Nguyen S.T., Ruoff R.S., Carbon, 45 (2007), 1558.

[25] Gao W., Alemany L.B., Ci L., Ajayan P.M., Nat. Chem., 1 (2009), 403.

[26] Mattevi C., Eda G., Agnoli S., Miller S., Mkhoyan K.A., CeliK O., Mastrogiovanni D., Granozzi G., Garfunkel E., ChHowalla M., Adv. Funct. Mater., 19 (2009), 2577.

Received 2015-05-27 Accepted 2016-07-21 